\title{
Material analysis for therapeutic insoles - A short report
}

\author{
Sathish Paul ${ }^{\mathrm{a}}$, David Prakash Kumar ${ }^{\mathrm{a}} \&$ B. Siva ${ }^{\mathrm{a}}$ \\ ${ }^{a}$ Schieffelin Institute of Health Research \& Leprosy Centre, Karigiri, \\ Vellore, Tamil Nadu, India
}

Submitted 8 January 2021; Accepted 26 January 2021

\begin{abstract}
Summary
Objective: Customized footwear with appropriate insole material for offloading is widely used for preventing ulceration in diabetic and leprosy affected patients.

Methods: Computer based finite element analysis was carried out on a 3 dimensionally modelled insole. The insole was tested with the boundary condition of constant geometry, constant loading and varied material properties to identify the biomechanical behaviour of material.

Results: A total of 7 commonly used insole rubber materials including Butyl Rubber, Ethylene Vinyl Acetate (EVA), Isoprene, Natural Rubber, Neoprene, Polyurethane and Micro Cellular Rubber (MCR) were tested using finite element analysis. The study exhibited low deformation and stress values for EVA. High deformation and stress values were seen in MCR.

Conclusion: The comparison of insole materials provides health workers with the required knowledge for selection of appropriate material for therapeutic insoles. The mechanical analysis of materials through the computer aided analysis will help the therapeutic footwear technician and designer to design, fabricate and use appropriate insoles.
\end{abstract}

Keywords: Diabetes, leprosy, therapeutic footwear, customized insoles, offloading

\section{Introduction}

The $0.5-3 \%$ of world's diabetic population develop plantar ulcers. Peak plantar pressures are identified as one of most common risk factors for plantar ulceration. ${ }^{1-3}$ Approximately $20 \%$ of diabetic patients with underlying foot infections may require amputation. ${ }^{4}$ High peak plantar pressures are common in the anaesthetic feet of patients affected with leprosy and other peripheral vascular diseases. Change in the foot structure due to repeated plantar ulceration can lead to further irregular distribution of plantar pressures. ${ }^{5}$ It has been established that the use of orthoses such as medial arch support, metatarsal bar and other customized insoles relieve

Correspondence to: Sathish Paul, Schieffelin Institute of Health Research \& Leprosy Centre, Karigiri, Vellore, Tamil Nadu, India (Tel.: +91 94423 79566; e-mail: sathishpaul77@ gmail.com) 
the pressure on the foot and prevent ulcers. ${ }^{6}$ Studies have also established that the reoccurrence of plantar ulceration could be prevented if the peak plantar pressure is maintained below 200 Kilopascals. ${ }^{7}$ Foot ulcers, caused by high peak plantar pressures can be significantly reduced through appropriate footwear and customized insoles. ${ }^{8}$ Though various materials are suitable to fabricate orthoses and footwear, the limitations in using the materials relate to the lack of durability and their high costs. Health staff and patients lack scientific evidence to identify and use ideal materials to fabricate orthotic insoles. Scientifically validated evidence on the changes and deformation in the material to the varied external forces would help clinicians and patients use appropriate materials for fabricating orthoses. Identifying materials that would be durable and cost effective while effectively dissipating the peak plantar pressures on the foot, is the need of the hour. Footwear with ideal insoles which dissipate the pressure are also essential for people who have deformed, ulcerated and anaesthetic feet. ${ }^{9}$ Materials such as Micro Cellular Rubber (MCR), Ethylene Vinyl Acetate (EVA) and Polyurethane are commonly used insole materials in fabricating appropriate footwear for various foot related problems. ${ }^{9-11}$ EVA and MCR are widely preferred and used in fabricating orthoses for leprosy as well as diabetic patients ${ }^{12}$.

The aim of this study was to compare the offloading properties of the commonly used materials in fabricating insoles. The study was also initiated to determine the effective and ideal insole materials that can be used in effectively dissipating peak plantar pressure. Earlier studies carried out by the author have demonstrated the effectiveness of analysing tissue stress using the finite element analysis (FEA) method. ${ }^{13}$ The present study used finite element analysis to establish the efficacy of the materials used in fabricating orthoses and foot insoles.

The FEA was carried out on an insole designed for a normal adult foot shape. The loading of the foot model was tested on the insole with varied material properties. Standardized properties like the yield stress, tensile strength and Young's modulus available in the literature were provided for the materials to carry out the computer based simulation. ${ }^{14,15}$ The material properties of Butyl Rubber, Ethylene Vinyl Acetate (EVA), Isoprene, Natural Rubber, Neoprene, Polyurethane and Micro Cellular Rubber (MCR) were compared and tested on the designed insole. The response of the materials to the simulation and its effect in dissipating peak plantar pressures were further analysed in this study.

\section{Materials and methods}

The insole for a normal adult foot and a realistic 3-dimensional (3D) model of a foot were designed using Computer Aided Designing (CAD) software. Finite Element Analysis (FEA) was used to study the interaction between the 3D foot model and the insole to evaluate the biomechanical behaviour of the identified insole materials. SOLIDWORKS 2018 software was used for designing the insole and ANSYS 2019 R1 was used to carry out finite element analysis and evaluate the mechanical properties of the insole.

An insole of $10 \mathrm{~mm}$ thickness was designed using CAD software (SOLIDWORKS). The designed insole model was then exported to the FEA software (ANSYS) to carry out simulations. Computed Tomography (CT) images of a foot in the subtalar neutral position were used to create a realistic foot model ideal for the foot insole. ${ }^{16}$ Then the foot-insole assembly was created on CAD and exported to ANSYS for simulation.

The biomechanical response of the foot model with the insole was analysed. On the imported assembly, static structural analysis was carried out by altering the material properties of the insole, including Young's modulus and Poisson's ratio. Analysis of the insoles made from Isoprene, Butyl Rubber, Polyurethane, Neoprene, Micro Cellular Rubber, Ethylene Vinyl 


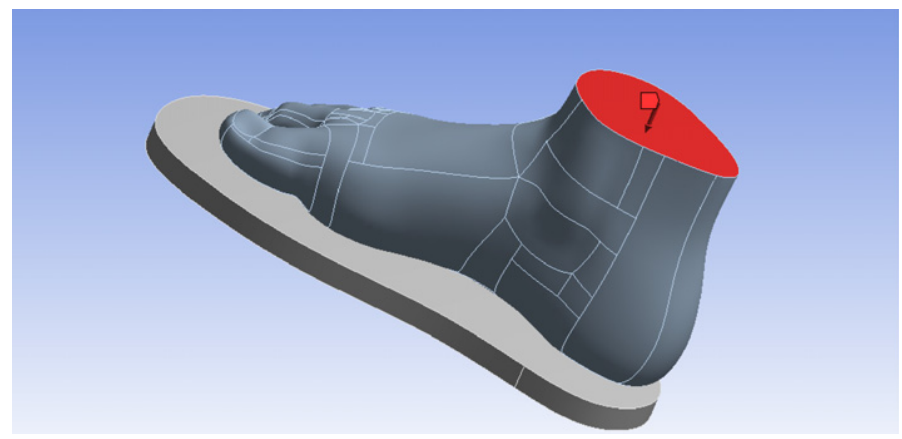

Figure 13 -Dimensional (3D) foot model for static structural analysis, with an insole with the properties of neoprene material.

Acetate and Natural Rubber were carried out through constant loading geometric and meshing properties. ${ }^{17}$ The mechanical properties, such as elasticity, stress, strain and deformation of each material were analysed on the designed insole.

The insole materials were simulated and tested for deformation by applying a force of $686.7 \mathrm{~N}(70 \mathrm{~kg})$ through the foot model on the insole as shown in Figure 1. Mechanical behaviour, occurrence of total deformation, equivalent elastic stress and strain distribution on the insole were analysed. Relationships between stress and strain were independent of time and loading properties. The elasticity of the material was exhibited by the complete and immediate recovery of the material to its original shape on removal of load. Evidence demonstrates that among the materials, the one with the lowest elastic modulus has the highest elastic property. ${ }^{18-20}$

\section{Results}

The deformation results of the insole materials were analysed, as shown in Figures 2, 3 and 4. The result showed that Micro Cellular Rubber exhibited maximum deformation when compared with other materials. The materials exhibited elastic deformability, linear stress and strain until the yield point. Greater deformation exhibited a greater displacement in the insole. $^{21}$

Each material was analysed for the stress by measuring the intensity of a reaction to the externally applied load. Its internal force acting perpendicular to a unit of area within the material was also analysed for stress. ${ }^{22}$ Mechanical properties, including stress, strain and deformation, represent the strength, resistance to failure, hardness, elasticity and shock absorption properties of the materials.

The analysis revealed that Ethylene Vinyl Acetate (EVA) material had the lowest stress concentration when compared to other materials. Hence EVA could provide greater stability to the foot when compared with other rubber materials.

\section{Discussion}

A total of seven materials were simulated and analysed in the study. The analysis established that among all the materials tested, EVA exhibited low stress value and MCR high deformation values. The lower deformation and the stress values exhibited by the EVA make it an ideal material for orthosis fabrication. The decreased resilience and the damping property of the 


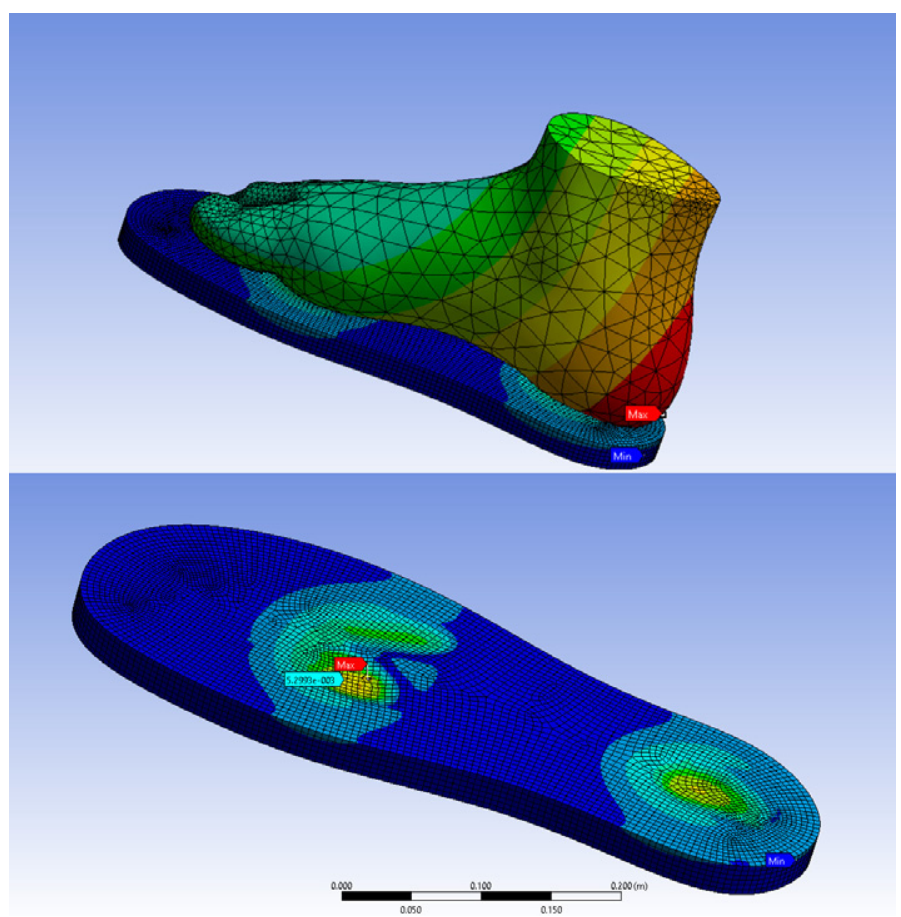

Figure 2 Simulation result of deformation on neoprene insole due to loading condition.

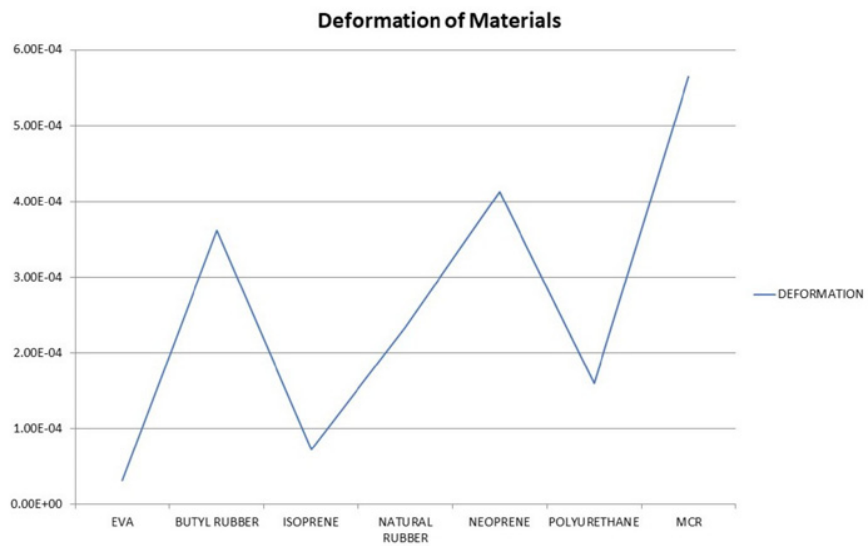

Figure 3 Deformation of the insole for various materials.

rubber makes it more stable and effective. ${ }^{23,24}$ The properties of the EVA makes it an ideal rubber for moulding and fabricating orthoses for the impaired foot. Results of earlier studies have shown similar results, in demonstrating that EVA has good shock absorption properties for a durable orthotic insole. ${ }^{25,26}$ Studies have established that a three layered sandal with MCR as the midsole, polyurethane foam as the insole, and EVA in its varied co-polymer densities to provide stability to the footwear as an outer sole would be ideal. ${ }^{5}$ Footwear 


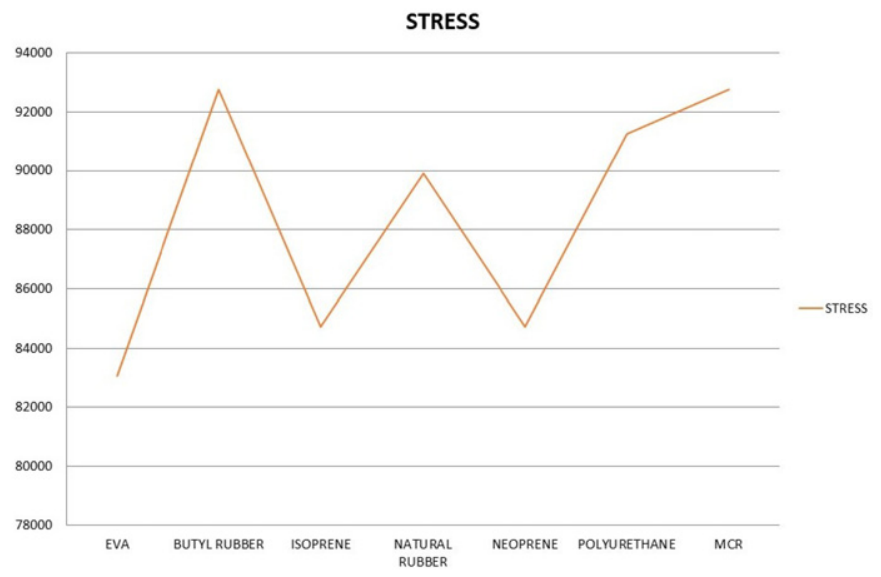

Figure 4 Graphical representation of stress formation on various materials.

consumption across the world has reached 24.2 billion in 2018 leading to the use of synthetic polymers and increasing environmental pollution. In order to overcome the problem, biodegradable material like Polyurethane (PU) could eventually replace the existing materials. Deproteinized natural rubber latex (DPNR) could also be used as a therapeutic insole with excellent pressure reduction and shock absorption properties. ${ }^{27}$

The increased deformation and the stress values of MCR makes it ideal to dissipate the pressure while walking and during other functional activities in leprosy affected patients. The elastic deformation property facilitates the MCR to offload the excessive peak plantar pressures and to reduce the shearing stresses in footwear. These properties of MCR rubber further reiterate the efficacy and durability of MCR footwear. Micro Cellular Rubber (MCR) insoles are widely used and are recommended for leprosy affected patients with anaesthetic feet. ${ }^{28}$ Studies have established that the lack of different designs and colours could decrease the acceptability of footwear among patients. ${ }^{29,30} \mathrm{MCR}$ insoles, if made in different colours without compromising on the properties established in the study, could increase the acceptance among the leprosy affected patients. The rubber materials analysed in this study are presently used as insoles and fabricated in varied combinations in the footwear industry. This study has also established that material properties of polyurethane are ideal in dissipating pressures and could be an alternative to MCR for anaesthetic feet.

Use of technology and technology based interventions have been established to be useful in improving compliance and adherence to treatments. ${ }^{31}$ Further studies on simulating the interactions between the foot and insoles would help in improving treatment outcomes and compliance in wearing footwear. Using the 'internet of things' and sensor technology would help in understanding the wear and tear on the insoles. It would also help in improving the quality of life of those affected by debilitating diseases like leprosy and diabetes. This simulation study will enable clinicians to identify the durability and the limitations of various orthotic material exposed to external forces.

\section{Conclusions}

The study establishes and reinforces the significance of rubber materials and their properties in offloading an anaesthetic foot. The study shows that the materials tested when used as 
insoles would significantly offload the foot with peak plantar pressures. However, the higher deformation characteristics exhibited by MCR would make it an ideal rubber to dissipate peak plantar pressures in footwear. The EVA with lower deformation characteristics could be effective in providing stability to the functional and adaptive orthoses fabricated for leprosy affected and other patients with foot impairments. The accessibility and affordability of MCR and EVA make them desirable offloading products for the anaesthetic foot.

\section{Declarations}

\section{Ethics approval and consent to participate}

Ethics review board review and approval was required for this study. All study procedures were approved by the Schieffelin Institute of Health Research and Leprosy Centre, ethics review board, approval number: 14:05:04.2.

\section{Conflict of interest statement}

The authors report no conflict of interest.

\section{Funding}

The authors would like to acknowledge the Leprosy Research Initiative (www.leprosyresearc h.org) for funding this project through the grant LRI.706.18.29/LRI.

\section{Authors contributions}

Dr. Sathish Kumar Paul (Corresponding author) contributes on Conceptualization, Data curation, Investigation, Supervision and validation.

Mr. David Prakash Kumar (Co-author1) contributes on Funding acquisition, Conceptualization, Data curation and Project Administration.

Mr. B. Siva (Co-author2) contributes on Formal analysis, Methodology, Software, Visualization and writing draft.

\section{Patient consent statement}

No patient consent was required.

\section{Acknowledgements}

The authors would like to acknowledge Dr. Jerry Joshua, Director, SIHRLC Karigiri for his valuable inputs and suggestions.

\section{References}

1 Sutkowska E, Sutkowski K, Sokołowski M et al. Distribution of the highest plantar pressure regions in patients with diabetes and its association with peripheral neuropathy, gender, age, and BMI: one centre study. J Diabetes Res, 2019; 2019: 7395769. doi:10.1155/2019/7395769.

2 Mohd A, Justine M, Manaf H. Plantar pressure distribution among older persons with different types of foot and its correlation with functional reach distance. Scientifica (Cairo), 2016; 2016: 8564020.

3 Yavuz M, Erdemir A, Botek G et al. Peak plantar pressure and shear locations: relevance to diabetic patients. Diabetes Care, 2007; 30(10): 2643-2645. doi:10.2337/dc07-0862.

4 Moral M, Martínez JL, Morales GE et al. Clinical efficacy of therapeutic footwear with a rigid rocker sole in the prevention of recurrence in patients with diabetes mellitus and diabetic polineuropathy: A randomized clinical trial. PLoS ONE, 2019; 14(7): e0219537. doi:10.1371/journal.pone.0219537. 
5 Viswanathan V, Madhavan S, Gnanasundaram S et al. Effectiveness of different types of footwear insoles for the diabetic neuropathic foot: a follow-up study. Diabetes Care, 2004; 27(2): 474-477. doi:10.2337/diacare.27. 2.474 .

6 Cross H, Sane S, Dey A et al. The efficacy of podiatric orthoses as an adjunct to the treatment of plantar ulceration in leprosy. Lepr Rev, 1995; 66(2): 144-157. doi:10.5935/0305-7518.19950016; PMID: 7637525.

7 Bus SA, Waaijman R, Arts M et al. Effect of custom-made footwear on foot ulcer recurrence in diabetes: a multicenter randomized controlled trial. Diabetes Care, 2013; 36(12): 4109-4116. doi:10.2337/dc13-0996.

8 Hellstrand Tang U, Zügner R, Lisovskaja V et al. Comparison of plantar pressure in three types of insole given to patients with diabetes at risk of developing foot ulcers - A two-year, randomized trial. J Clin Transl Endocrinol, 2014; 1(4): 121-132. doi:10.1016/j.jcte.2014.06.002.

9 Lehman LF, Geyer MJ, Bolton L. American leprosy missions use of protective footwear. In: Ten Steps: A Guide for Health Promotion and Empowerment of People Affected by Neglected Tropical Diseases. USA: American Leprosy Missions, 2015; pp. 175-187.

10 Cross H. Wound Care for People Affected by Leprosy A Guide for Low Resource Situation. American Leprosy Mission, 2003.

11 Park C, Choi W, Lee J. Effects of hardness and thickness of polyurethane foam midsoles on bending properties of the footwear. Fibers Polym, 2007; 8: 192-197.

12 Govindasamy K, Raja NR, Gupta P, Anand G, Das P, Darlong F, Darlong J. Computer assisted customized footwear and traditional micro-cellular rubber (MCR) footwear to reduce recurrence of ulcer for patients with loss of sensation due to leprosy. Lepr Rev, 2020; 91(4): 383-392. doi:10.47276/1r.91.4.383.

13 Paul SK, Vijayakumar R, Mathew L et al. Finite element model-based evaluation of tissue stress variations to fabricate corrective orthosis in feet with neutral subtalar joint. Prosthet Orthot Int, 2017; 41(2): 157-163.

14 Materials Data Book, 2003, Edition Cambridge University Engineering Department.

15 Hanhi K, Poikelispaa M, Tirila HM. Elastometric materials. Plast Elastomer Technol, 2007; 1-84.doi: 10.1051/orthodfr/2008030.

16 Paul SK, Rekha VK, Sivarasu S. Customized Insole fabrication for foot deformities in Leprosy patients. J Med Devices, 2014; 8(2): 020950. doi:10.1115/1.4027065.

17 Roylance D. Mechanical Properties of Materials, Material Science, Corpus ID: 137567769. 2008. doi:10.120 1/9781420061345-12.

18 Kumar P, Moumita et al. An Overview of Stress-Strain Analysis for Elasticity Equations, Elasticity of Materials - Basic Principles and Design of Structures, 2018, Ezgi Günay, IntechOpen, doi:10.5772/intechopen.82066.

19 Abdelgader H, Jarosław . Stress-strain relations and modulus of elasticity of two-stage concrete. J Mater Civil Eng, 2003; 15(4): doi:10.1061/(ASCE)0899-1561(2003)15:4(329).

20 Lo WT, Yick KL, Ng SP et al. Numerical simulation of orthotic insole deformation for diabetic foot. $J$ Fiber Bioeng Inform, 2005; 8: 401-411.

21 Rees D. Basic Engineering Plasticity: An Introduction with Engineering and Manufacturing Applications. Boston, MA: Elsevier, 2006; pp. 1-318.

22 Brito e Dias R, Pena Coto N, Ferreira Batalha G, Driemeier L. Systematic Study of Ethylene-Vinyl Acetate (EVA) in the Manufacturing of Protector Devices for the Orofacial System. In: Dobrzański LA (ed.), Biomaterials in Regenerative Medicine. IntechOpen, 2018. doi:10.5772/intechopen.69969.

23 Kim M-S, Park C-C, Chowdhury SR, Kim G-H. Physical properties of ethylene vinyl acetate copolymer (EVA)/natural rubber (NR) blend based foam. J Appl Polym Sci, 2004; 94(5): 2212-2216. doi:10.1002/app.21174

24 Wang L, Hong Y, Li JX. Durability of running shoes with ethylene vinyl acetate or polyurethane midsoles. J Sports Sci, 2012; 30(16): 1787-1792.

25 Healy A, Dunning DN, Chockalingam N. Effect of insole material on lower limb kinematics and plantar pressures during treadmill walking. Prosthet Orthot Int, 2012; 36(1): 53-62. doi:10.1177/0309364611429986.

26 Mukherjee, Thangavelu, Chelike et al.Biodegradable polyurethane foam as shoe insole to reduce footwear waste: Optimization by morphological physicochemical and mechanical properties. Appl Surf Sci, 2020; 499: 143966.

27 Ramli , Rubaizah, Fatimah . Novel deproteinised natural rubber latex slow-recovery foam for health care and therapeutic foam product applications. J Rubber Res, 2018; 21(2018): 277-292.

28 Paul SK, Rajkumar E, Mendis T. Micro Cellular Rubber (MCR) - a boon for leprosy affected patients with anesthetic feet in preventing secondary impairments. J Foot Ankle Res, 2014; 7: A92.

29 Karthikeyan GP, Nathan RJ. Footwear for the person with an anesthetic foot: What options are available? Lepr Rev, 2017; 2017(88): 265-269.

30 Govindharaj P, Suresh, Joydeepa et al. Acceptance and satisfaction of micro-cellular rubber ready-made footwear among patients with insensitive feet due to leprosy. Lepr Rev, 2017; 88: 381-390.

31 Paul SK, Kumar DP. Use of mobile technology in preventing leprosy impairments. Disabil Rehabil Assist Technol, 2020; 1-3. 